\title{
Transverse electric surface waves in ferrite medium surrounded by plasma layers
}

\author{
M. Umair ${ }^{1}$, A. Ghaffar ${ }^{1 *}$, Majeed A. S. Alkanhal ${ }^{2}$, Ali H. Alqahtani ${ }^{3}$ and Y. Khan²
}

\begin{abstract}
The theoretical analysis of transverse electric surface waves in ferrite medium surrounded by isotropic plasma layers is presented in this manuscript. Maxwell's equations in differential form are used, and we impose the boundary conditions to acquire the dispersion relation to formulate the proposed structure. The influence of number density, separation distance between the layers of plasma, and dielectric permittivity of ferrite film on the normalized propagation constant is studied. It is concluded from the result obtained that if the number density and values of dielectric permittivity of ferrite film increases the propagation constant $\operatorname{Re}(\beta)$ tends to decreases whereas the increase in separation distance between the layers of plasma tends to increase the propagation constant $\operatorname{Re}(\beta)$. Furthermore, to verify the surface waves, the normalized field distribution for plasma medium as well as ferrite medium are also presented in this manuscript. The present work has potential applications in communication, drug delivery, cancer treatment, and ferrite sensing waveguide structures in the $\mathrm{GHz}$ frequency regime.
\end{abstract}

\section{Introduction}

Investigation of the electromagnetic traits of plasma waveguide structures reveals attractive features that are very significant for potential applications in communication, cancer treatment, advancement in methods of charge particle acceleration, transport of high current charge particle beams, and generation of high-power electromagnetic radiation [1-3]. Theoretical and plasma physicists categorize plasma as anisotropic plasma and isotropic plasma. Anisotropic plasma is direction dependent and has an external magnetic field, while in the case of isotropic plasma, there is no external magnetic field and it has the same properties in all directions. Isotropic plasma supports highfrequency electromagnetic waves, which see the plasma as a simple dielectric owing to the response of free electrons to the wave. In the case of cold plasma, these waves exhibit simple oscillation at the plasma frequency, which is the function of number density, below which electromagnetic waves do not propagate.

Recently, ferrite materials have attracted considerable attention in different areas of nanotechnology research.

\footnotetext{
*Correspondence: aghaffar16@uaf.edu.pk

'Department of Physics, University of Agriculture, Faisalabad, Pakistan

Full list of author information is available at the end of the article
}

Ferrite is an anisotropic magnetic material having superior fabrication and design properties widely used in passive and Antenna microwave devices such as filters, circulators, phase shifters, and isolators [4-11]. Since ferrite magnetic tensors can be altered by tailoring the DC magnetic bias, ferromagnetic materials are used to design many reconfigurable electromagnetic devices. Due to their adjustable magnetic traits, ferrite materials are widely used for designing the latest reconfigurable antennae [12]. These traits motivate us to study the ferrite-filled waveguide, which has not yet been studied. Many researchers carried out research on surface wave and wave propagation for waveguides [13-19].

The presented work of this manuscript deals with a waveguide made of a plasma-ferrite-plasma planar interface in the $\mathrm{GHz}$ operating surface wave frequency. In this work, we present the analysis and computation of the propagation constant and field distribution. The dispersion relations for the guided TE surface waves are derived and used to acquire the propagating mode and normalized field distribution. The influence of the number density of electrons, separation distance, and dielectric permittivity of the ferrite material on the propagation constant is studied. 
Moreover, the normalized field distribution for plasma and ferrite medium is also presented in this manuscript.

\section{Geometry and equations}

The geometric configuration of the proposed dielectric/ ferrite/dielectric sandwich structure for the propagation of the guided TE surface waves is presented in Fig. 1. The structure consists of ferrite medium bounded by isotropic plasma materials. We assume that the electric and magnetic field components are proportional to $e^{i(k z-}$ $\omega t)$ and $\mathrm{y}$ independent $i . e . \partial / \partial y=0$ where $\omega$ is the operating surface wave frequency and $\mathrm{k}$ is the propagation vector in the $\mathrm{z}$ direction. To obtain uniform intensity $H_{0}$ within the ferrite film, we apply static magnetic field $B_{0}$ along the y direction. The electric and magnetic field components for the propagation of TE surface waves along the $\mathrm{z}$ for the presented geometry have the following form as presented in [20].

$$
\begin{aligned}
& E=\left[0, E_{y}, 0\right] e^{i\left(k_{z}-\omega t\right)} \\
& H=\left[H_{x}, 0, H_{z}\right] e^{i\left(k_{z}-\omega t\right)} .
\end{aligned}
$$

Field component for plasma medium $(x<0)$ and $(d<x)$

We consider that a plasma medium with region $1(x<0)$ and with region $3(d<x)$ as shown in Fig. 1 . By assuming the time-dependent perturbations, the momentum transfer, equation of continuity and Maxwell's equations can be used to express the plasma medium in the absence of electric field and an equilibrium drift velocity [21, 22]:

$$
\begin{aligned}
& n m(-i \omega \boldsymbol{V}+v \boldsymbol{V})=-e n \boldsymbol{E}-\nabla \mathrm{p} \\
& i \omega p=n m v_{t h}^{2} \nabla \cdot \mathbf{V} \\
& \nabla \times \boldsymbol{H}=-e n \boldsymbol{V}-i \omega \varepsilon_{0} \boldsymbol{E} \\
& \nabla \times \boldsymbol{E}=i \omega \mu_{0} \boldsymbol{H}
\end{aligned}
$$

Where $n, m, v, V, e, \mathrm{p}$ and $v_{t h}=\sqrt{K T \gamma / m}$ are electron number density, mass of electron, collision frequency, velocity, charge on electron, pressure and thermal velocity respectively whereas $K, T$ and $\gamma$ are Boltzmann constant, temperature of plasma and ratio of specific heat. By using value of $\boldsymbol{V}$ in equation (4) from equations $(2,3)$ we get

$$
\nabla(\nabla . \mathbf{E})-\nabla^{2} \boldsymbol{E}=\frac{\omega^{2}}{c^{2}}\left[\left(1-\frac{\omega_{p}^{2}}{\omega^{2}+i \omega \nu}\right) \boldsymbol{E}+\left(\frac{v_{t h}^{2}}{\omega^{2}+i \omega \nu}\right) \nabla(\nabla . \mathbf{E})\right]
$$

where plasma frequency is defined as $\omega_{p}=\left(e^{2} n / \varepsilon_{0} m\right)^{\frac{1}{2}}$ In our proposed model the electric field $\boldsymbol{E}=E_{y} \hat{j}$ and $\nabla$. $\mathbf{E}=(\hat{i} \partial / \partial \mathrm{x}+\hat{k} \partial / \partial \mathrm{z}) \cdot\left(E_{y} \hat{j}\right)=0$. The equation (6) becomes as presented in [20].

$$
\frac{d^{2} E_{y}}{d x^{2}}-k_{p}^{2} E_{y}=0
$$

where $k_{p}^{2}=\beta^{2}-k_{0}^{2} \varepsilon_{p}$ with propagation constant $\beta, k_{0}^{2}$ $=\omega^{2} / c^{2}$ and $\varepsilon_{p}=1-\frac{\omega_{p}^{2}}{\omega^{2}+i \omega \nu}$.

The solution of equation (5) for region $1(x<0)$ and region $3(d<x)$ are given below respectively

$$
E_{y 1}=C e^{k_{p} x} .
$$

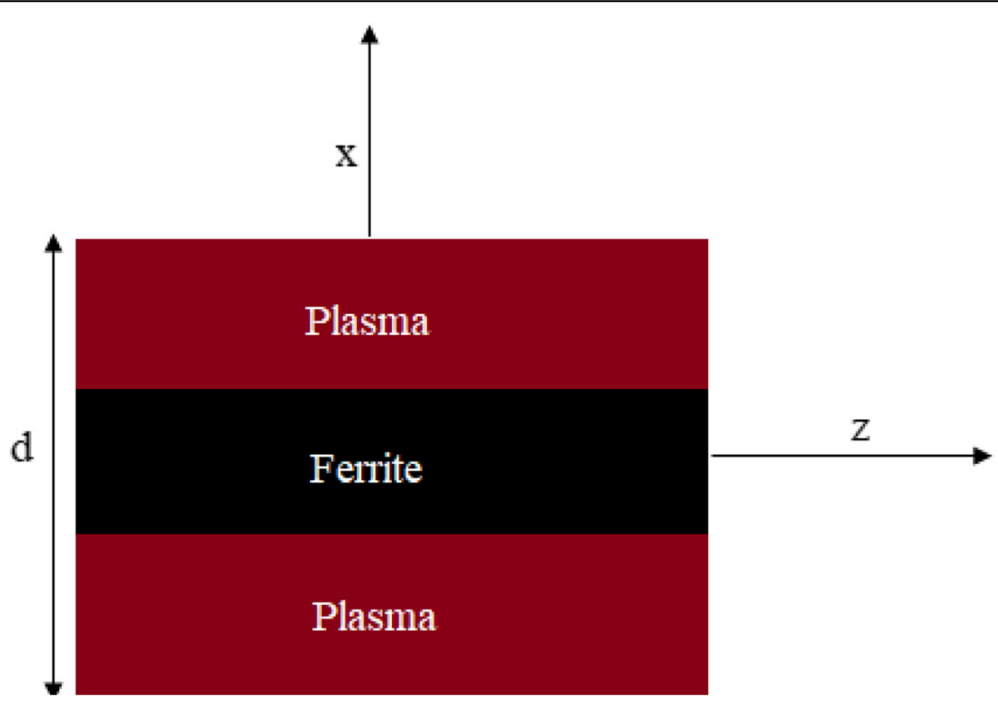

Fig. 1 Geometry for plasma parallel plate waveguide filled with ferrite 


$$
E_{y 3}=D e^{-k_{p} x}
$$

where $\mathrm{C}$ and $\mathrm{D}$ are unknown constants.

Field component for ferrite medium $(0<x$ and $<x)$ In this structure, a ferrite film is sandwiched between plasma materials. For this region, Maxwell's equations can be written as

$$
\begin{aligned}
& \nabla \times \boldsymbol{H}=-i \omega \varepsilon_{0} \varepsilon_{f} \boldsymbol{E} \\
& \nabla \times \boldsymbol{E}=i \omega \mu_{0} \bar{\mu} \boldsymbol{H} .
\end{aligned}
$$

The model field for ferrite medium is

$$
E_{y 2}=A \cosh \left(k_{f} x\right)+B \sinh \left(k_{f} x\right)
$$

where A, B are unknown constants, $k_{f}=\sqrt{\beta^{2}-k_{0}^{2} \varepsilon_{f} \mu_{v}}$ is wave number in ferrite films, $\mu_{v}=\mu_{x x}{ }^{2}-\mu_{z x} / \mu_{x x}$ is called Voigt permeability as presented in [20] and $\varepsilon_{f}$ is the dielectric constant for the ferrite material. The $\bar{\mu}$ is the Polder permeability tensor as:

$$
\bar{\mu}=\left(\begin{array}{ccc}
\mu_{x x} & 0 & i \mu_{z x} \\
0 & \mu_{y y} & 0 \\
-i \mu_{z x} & 0 & \mu_{x x}
\end{array}\right) .
$$

Here, $\quad \mu_{x x}=\left(1+\frac{\omega_{m} \omega_{0}}{\omega_{0}^{2}-\omega}\right) \mu_{b}, \quad \mu_{z x}=\left(1+\frac{\omega_{m} \omega}{\omega_{0}^{2}-\omega}\right) \mu_{b}$, and $\mu_{y y}=\mu_{b}$ are the Polder tensor elements; $\mu_{b}$ is the optical magnon permeability; and $\omega_{m}$ and $\omega_{0}$ are given as $\omega_{m}=\mu_{0} \gamma M_{0}, M_{0}$ is the static magnetization and $\omega_{0}=$ $\mu_{0} \gamma H_{0}$. Here, $H_{0}$ is the uniform intensity within the ferrite film resulting from the applied static magnetic field $B_{0}$ and $\gamma$ is the gyromagnetic ratio [23, 24]. Owing to the anisotropy of the ferrite material, the analysis of

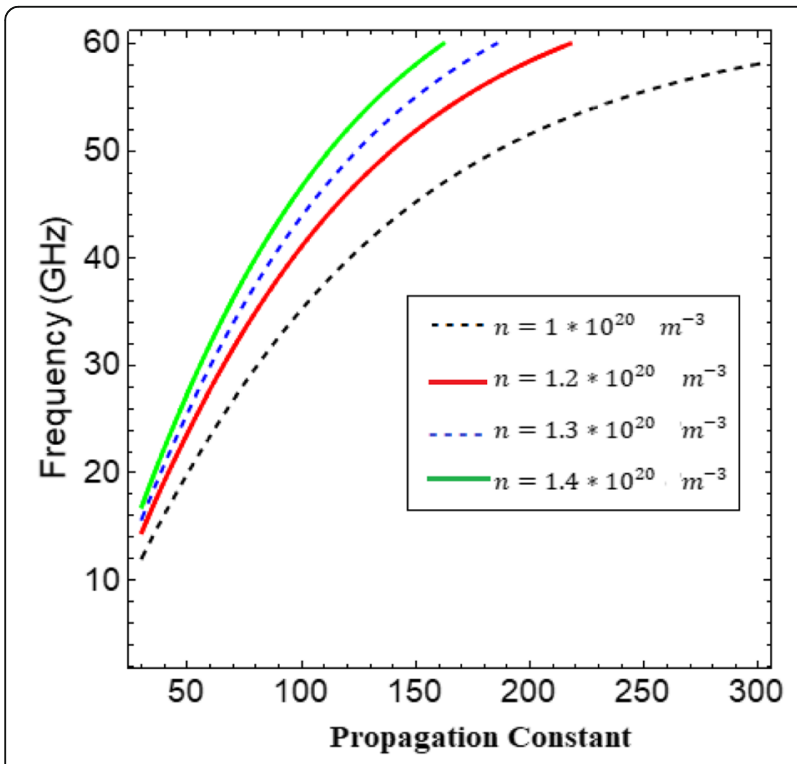

Fig. 2 Influence of number density on propagation constant

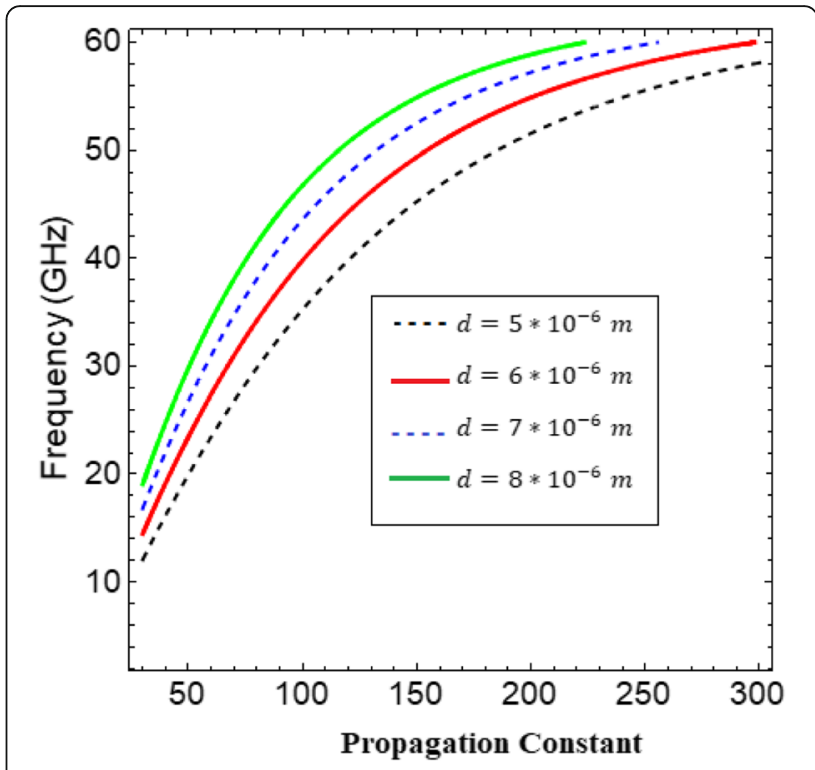

Fig. 3 Influence of plate separation on propagation constant

the ferrite waveguide is complicated. The following boundary conditions are used to solve the proposed structure:

$$
\begin{aligned}
& \hat{\boldsymbol{x}} \times\left(\boldsymbol{H}_{2}-\boldsymbol{H}_{\mathbf{1}}\right)=\mathbf{0} \\
& \hat{\boldsymbol{x}} \times\left(\boldsymbol{E}_{2}-\boldsymbol{E}_{\mathbf{1}}\right)=\mathbf{0}
\end{aligned}
$$

By applying these boundary conditions at the plasmaferrite and ferrite-plasma interfaces following matrix is yielded as:

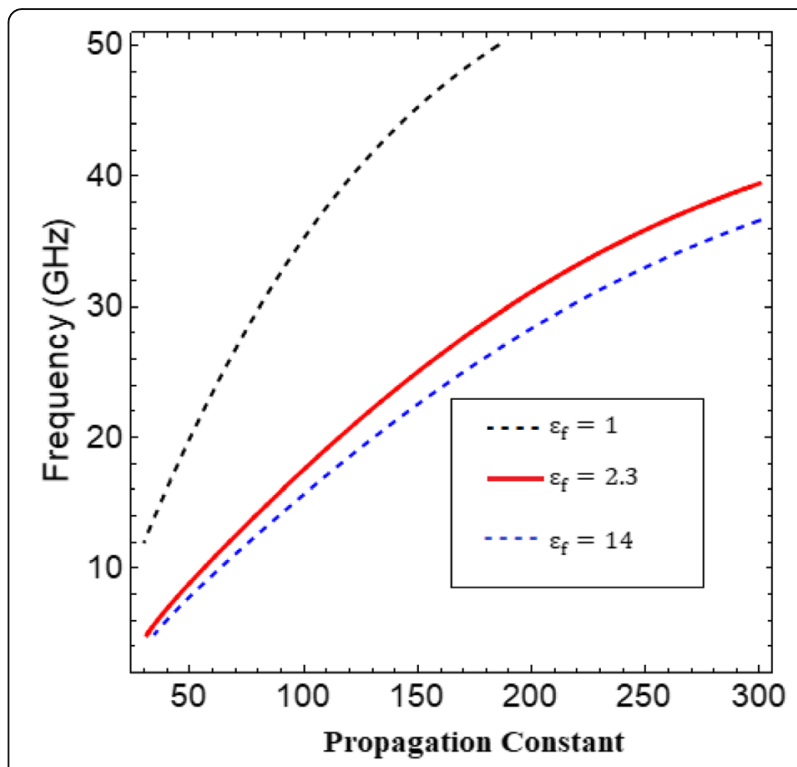

Fig. 4 Influence of dielectric permittivity of ferrite material on propagation constant 

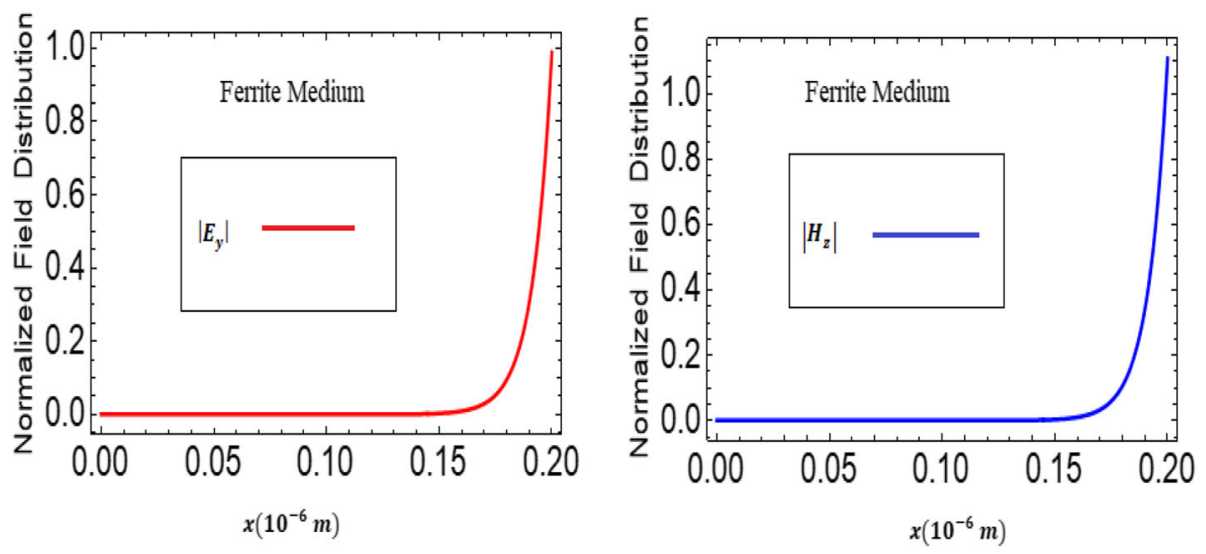

Fig. 5 Normalized field distribution of ferrite medium

$$
\left[\begin{array}{cccc}
a_{11} & a_{12} & a_{13} & a_{14} \\
a_{21} & a_{22} & a_{23} & a_{24} \\
a_{31} & a_{32} & a_{33} & a_{34} \\
a_{41} & a_{42} & a_{43} & a_{44}
\end{array}\right]\left[\begin{array}{c}
A \\
B \\
C \\
D
\end{array}\right]=0
$$

The entities in matrix are defined as $a_{11}=1, a_{12}=0$, $a_{13}=-1, a_{14}=0, a_{21}=0, a_{22}=-\frac{i k_{f}}{\varepsilon_{f} \mu_{0}}, a_{23}=\frac{i k_{p}}{\varepsilon_{p} \mu_{0} \omega}, a_{24}=$ $0, a_{31}=-\cosh \left(d k_{f}\right), a_{32}=\sinh \left(d k_{f}\right), a_{33}=0, a_{34}=e^{-k_{p} d}$, $a_{41}=\frac{i k_{f} \sinh \left(d k_{f}\right)}{\varepsilon_{f} \mu_{0} \omega}, \quad a_{42}=\frac{i k_{f} \cosh \left(d k_{f}\right)}{\varepsilon_{f} \mu_{0} \omega}, a_{43}=0$ and $a_{44}$ $=\frac{i e^{-k p d} k_{p}}{\varepsilon_{p} \mu_{0} \omega}$,

After solving the matrix, we get dispersion relation:

$$
\operatorname{Tanh}\left[d \mathrm{k}_{f}\right]+\frac{2 \mathrm{k}_{f} \mathrm{k}_{p} \varepsilon_{f} \varepsilon_{p}}{\left(\left(\mathrm{k}_{p} \varepsilon_{f}\right)^{2}+\left(\mathrm{k}_{f} \varepsilon_{p}\right)^{2}\right)}=0
$$

\section{Results and discussion}

The derived characteristic equation for the plasmaferrite-plasma structure was computed numerically. The numerically simulated results are presented in graphical form using the computer software MATHEMATICA. In all the graphical results, the separation distance is kept at $d=10^{-6} \mathrm{~m}$. We can numerically illustrate the normalized propagation constant $\operatorname{Re}(\beta)$ with respect to the operating surface wave frequency $\omega$ for the different values of plasma frequency, separation distance $d$, and dielectric constant of ferrite film $\varepsilon_{f}$. The ferrite film parameter values are $\varepsilon_{f}=1, \quad \omega_{m}=3.08 * 10^{10} \mathrm{~Hz}, \quad \omega_{0}=0.343 *$ $10^{10} \times \omega_{m} \mathrm{~Hz}$, and $\mu_{b}=1$. We have assumed that $(v /$ $\omega)<<1$. Since guided TE surface waves are interlinked with $H_{x}$ and $H_{z}$, they depend upon the frequencydependent $\mu_{x x}$ and $\mu_{z x}$ components of the Voigt permeability, and its function is given as $\mu_{v}=\left(\mu_{x x}^{2}-\mu_{z x}^{2}\right) / \mu_{x x}$.

Fig. 2 illustrates the computed dispersion curve of TE surface waves for the different values of number densities i.e., $n=1 * 10^{20} \mathrm{~m}^{-3}$ (black dashed line), $n=1.2 *$ $10^{20} \mathrm{~m}^{-3}$ (red thick line), $n=1.3 * 10^{20} \mathrm{~m}^{-3}$ (blue dashed line) and $n=1.4 * 10^{20} \mathrm{~m}^{-3}$ (green thick line). Since the electron plasma frequency can be tuned by the number
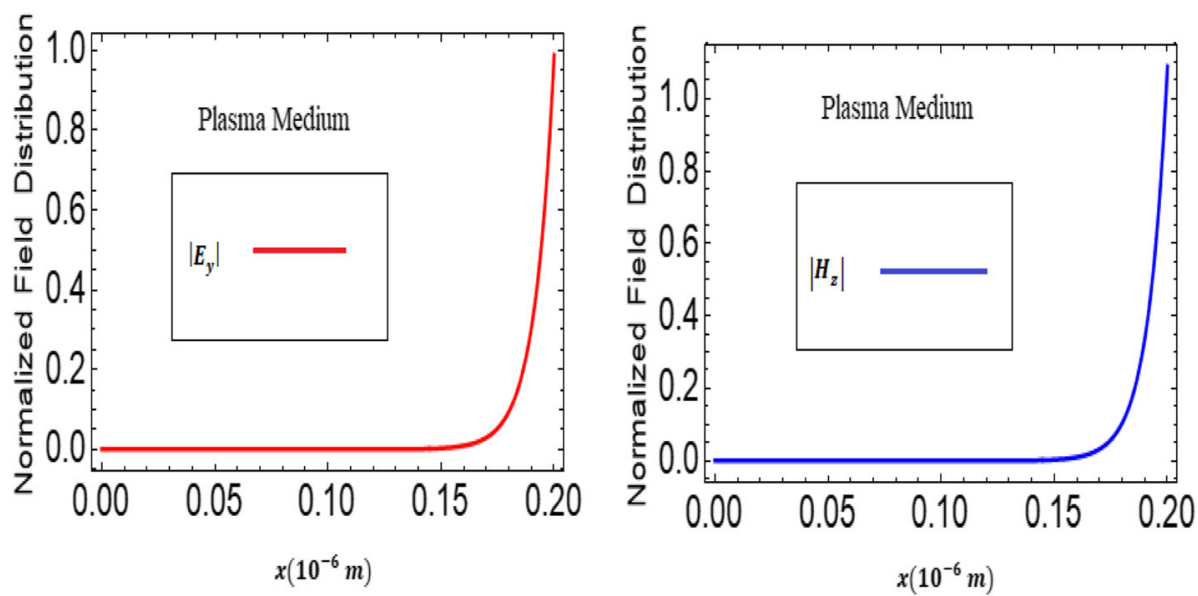

Fig. 6 Normalized field distribution of plasma medium 
density of free electrons, in Fig. 2, the propagation constant $\operatorname{Re}(\beta)$ with respect to the operating surface wave frequency $\omega$ in the $\mathrm{GHz}$ range is analyzed using the dispersion relation of equation (17). We have assumed over dense plasma material due its importance in theoretical as well experimental researches. The typical values of number density of electron are assumed to obey the condition of over dense plasma regime for $\omega<\omega_{p}$ when $\varepsilon_{p}$ $=1-\frac{\omega_{p}^{2}}{\omega^{2}}=1-\frac{\left(e^{2} n / \varepsilon_{0} m\right)}{\omega^{2}}<0$. It can be observed that as the number density of electrons increases, the normalized phase constant tends to decrease and peaks are shifted toward the higher frequency region in a certain frequency range as reported in [20]. As the propagation constant $\operatorname{Re}(\beta)$ decreases, the band gap also decreases, and thus, the band gap has potential practical applications for waveguide devices. It is concluded that the number density of electrons controls the surface wave propagation in the plasma-ferrite-plasma structure.

To study the confinement of guided surface waves, which is a significant parameter for the practical aspects of the proposed geometry, the normalized propagation constant $\operatorname{Re}(\beta)$ with respect to the operating surface wave frequency using the dispersion relation of equation (17) under the different values of plate separation i.e., $d=5$ * $10^{-6} \mathrm{~m}$ (black dashed line), $d=6 * 10^{-6} \mathrm{~m}$ (red thick line), $d=7 * 10^{-6} \mathrm{~m}$ (blue dashed line), and $d=8 * 10^{-6} \mathrm{~m}$ (green thick line) is presented in Fig. 3. It is obvious that as the separation distance (distance between the layers of plasma) can be varied by considerably varying the separation distance. It is noted that if separation distance plasma layer increases the normalized phase constant decreases as expected to obey the condition of over dense plasma. The numerical calculations are performed at the different values of dielectric permittivity of ferrite film i.e., $\varepsilon_{f}=1$ (black dashed line), $\varepsilon_{f}=2.3$ (red thick line) and $\varepsilon_{f}=$ 14 (blue dashed line) with respect to the operating surface wave frequency for the plasma-ferrite-plasma structure is analyzed in Fig. 4. As dielectric permittivity of ferrite film increases, the propagation constant $\operatorname{Re}(\beta)$ increases due to dielectric constant property of ferrite film and peaks shift toward the low-frequency region. Moreover, by decreasing the dielectric permittivity of ferrite, the band gap increases, which is very useful for the waveguide community. Representative modal fields are shown in Figures 5 and 6 of ferrite medium and plasma medium respectively for the plasma-ferrite-plasma structure with $d=5 * 10^{-6} \mathrm{~m}$ and $n=1 * 10^{20} \mathrm{~m}^{-3}$. Figures 5 and 6 illustrate the normalized field distribution of ferrite medium and plasma medium respectively for $\mathrm{TE}$ surface wave components $E_{y}$ and $H_{z}$. The surface wave has two conditions: (1) it moves away from the interface and (2) it undergoes exponential decay. Therefore, these simulations results verify the surface wave conditions.

\section{Conclusion}

We presented an analysis of guided TE surface waves for a ferrite medium surrounded by plasma layers. The dispersion relation was computed by applying the boundary conditions approach, and the following conclusions were drawn:

(i) Surface waves propagate along the plasma-ferriteplasma interface.

(ii) The normalized propagation constant is very sensitive to the number density of free electrons, which is the key parameter of isotropic plasma, dielectric permittivity of ferrite film, and separation distance of plasma layers.

(iii) To verify the surface waves, the normalized field distribution of plasma and ferrite medium was also discussed.

(iv) The presented work has potential applications in cancer treatment, communication, drug delivery, and ferrite-based waveguide devices in the $\mathrm{GHz}$ frequency range.

\section{Acknowledgements}

Not applicable.

\section{Authors' contributions}

Muhammad Umair, A. Ghaffar, derived analytical expressions and numerical analysis. They wrote the main manuscript text. Majeed Alkanhal, Ali H. Qahtani and Y.Khan developed methodology in the given study. The author(s) read and approved the final manuscript.

\section{Funding}

Deanship of Scientific Research (DSR) at King Saud University for its funding of this research through the Research Group no RG-1436-012.

Availability of data and materials

Detail about data has been provided in the article.

Competing interests

All authors contributed equally in all the sections of this work.

\section{Author details}

'Department of Physics, University of Agriculture, Faisalabad, Pakistan. ${ }^{2}$ Department of Electrical Engineering, King Saud University, Riyadh, Saudi Arabia. ${ }^{3}$ Department of Electrical Engineering, College of Applied Engineering, King Saud University, Al-Muzahmiah Branch, Al-Muzahmiah, Saudi Arabia.

Received: 31 January 2020 Accepted: 11 June 2020

Published online: 08 July 2020

References

1. Swanson, D.G., Plasma waves. CRC Press, Boca Raton (2003)

2. Vandenplas, P.E.: Electron Waves and Resonances in Bounded Plasmas (1968)

3. Zaginaylov, G., Shcherbinin, V., Shuenemann, K.: On the dispersion properties of waveguides filled with a magnetized plasma. Plasma physics reports. 31(7), 596-603 (2005)

4. Harris, V.G., et al.: Recent advances in processing and applications of microwave ferrites. J. Magn. Magn. Mater. 321(14), 2035-2047 (2009)

5. Kodera, T., Caloz, C.: Integrated leaky-wave antenna-duplexer/diplexer using CRLH uniform ferrite-loaded open waveguide. IEEE Trans. Antennas Propag. 58(8), 2508-2514 (2010) 
6. Kumar, D., Pourush, P.: Circular patch microstrip array antenna on NiCoAl ferrite substrate in C-band. J. Magn. Magn. Mater. 322(9-12), 1635-1638 (2010)

7. Laur, V., et al.: Application of molded interconnect device technology to the realization of a self-biased circulator. J. Magn. Magn. Mater. 404, 126-132 (2016)

8. Liu, J.-X., et al.: Anisotropic ferrite microstrip antenna simulation and analysis. Optik. 127(8), 4144-4149 (2016)

9. Stergiou, C.A., Litsardakis, G.: Y-type hexagonal ferrites for microwave absorber and antenna applications. J. Magn. Magn. Mater. 405, 54-61 (2016)

10. Wang, J., et al.: Design and simulation of self-biased circulators in the ultra high frequency band. J. Magn. Magn. Mater. 324(6), 991-994 (2012)

11. Zhang, Z., et al.: Microwave bandpass filters tuned by the magnetization of ferrite substrates. IEEE Magn. Lett. 8, 1-4 (2016)

12. Mohammadi, M., Kashani, F.H., Ghalibafan, J.: A partially ferrite-filled rectangular waveguide with CRLH response and its application to a magnetically scannable antenna. J. Magn. Magn. Mater. 491, 165551 (2019)

13. Abadla, M., Taya, S.A.: Excitation of TE surface polaritons in different structures comprising a left-handed material and a metal. Optik-Int. J Light Electron Optics. 25, 1401-1405 (2014)

14. Taya, S.A.: P-polarized surface waves in a slab waveguide with left-handed material for sensing applications. J. Magn. Magn. Mater. 377, 281-285 (2015)

15. Taya, S.A., Theoretical investigation of slab waveguide sensor using anisotropic metamaterial. Opt. Appl., 45(3), 405-417 (2015)

16. Taya, S.A., et al.: Slab waveguide with conducting interfaces as an efficient optical sensor: TE case. J. Mod. Opt. 64(8), 836-843 (2017)

17. El-Amassi, D.M., Taya, S.A.: Reflection through a parallel-plate waveguide formed by two graphene sheets. Photonics and NanostructuresFundamentals and Applications. 24, 53-57 (2017)

18. Taya, S.A., et al.: Photonic crystal with epsilon negative and double negative materials as an optical sensor. Opt. Quant. Electron. 50(5), 222 (2018)

19. Yaqoob, M.Z., et al.: Characteristics of light-plasmon coupling on chiralgraphene interface. JOSA B. 36(1), 90-95 (2019)

20. Ali, R., Zamir, B., Shah, H.: Transverse electric surface waves in a plasma medium bounded by magnetic materials. Results in physics. 8, 243-248 (2018)

21. Ghosh, S., Pal, S.: A study of plasma filled parallel plate waveguide with one boundary corrugated. Czechoslovak J. Physics B. 31(1), 25-31 (1981)

22. Shaikh, M., Zamir, B., Ali, R.: TE surface waves in a plasma sandwich structure. Acta Phys. Pol. A. 127(6), 1625-1629 (2015)

23. Boardman, A., Shabat, M.M., Wallis, R.: Nonlinear magnetodynamic waves on magnetic materials. Phys. Rev. B. 41(1), 717 (1990)

24. Hartstein, A., et al.: Surface polaritons on semi-infinite gyromagnetic media. J. Phys. C Solid State Phys. 6(7), 1266 (1973)

\section{Publisher's Note}

Springer Nature remains neutral with regard to jurisdictional claims in published maps and institutional affiliations.

\section{Submit your manuscript to a SpringerOpen ${ }^{\circ}$ journal and benefit from:}

- Convenient online submission

- Rigorous peer review

- Open access: articles freely available online

- High visibility within the field

- Retaining the copyright to your article

Submit your next manuscript at $\boldsymbol{\nabla}$ springeropen.com 